\title{
MTF Estimation Based on an Improved Step edge Method
}

\author{
Mengya $\mathrm{Qu}^{1,}$, , Yongsheng Zhang $^{1}$, and Weican Meng ${ }^{2}$ \\ ${ }^{1}$ Institute of Surveying and Mapping, Zhengzhou 450000, China; \\ ${ }^{2}$ Beijing Institute of Tracking and Telecommunication Technology, Beijing 100000, China. \\ ameng20041126@163.com
}

Keywords: Image processing, Modulation Transfer Function (MTF), step edge method.

\begin{abstract}
A higher precision reconstruction method for MTF, not constrained by the slant edge angle, is proposed. The improved method adopts new techniques of gradient operator and moving window in ESP sample refinement to gain more accurate and robust MTF estimated results. Experiments show that the new method is considerably superior in accuracy and stability at different edge angles.
\end{abstract}

\section{Introduction}

The MTF (Modulation Transfer Function) is a means of evaluating and quantifying the quality of the optical imaging system, reflecting its capability to contrast and to discern objects' detailed information. According to different signal patterns, three common methods can be used to estimate the absolute MTF: step edge method [1], point source method [2], and rectangular pulse method [3]. Since the step edges are easy to find in practice, the step edge method is the most widely adopted one.

In the research of image quality assessment of AVNIR-2 and ALOS PRISM, it is found during calculation of the absolute MTF that non-uniform distribution of ESF sample points and nonlinear noise on low and high radiance regions make it crucial to select the suitable ESF fitting curve [4]. Therefore, in this article, an improved step edge method is proposed to measure the MTF with consideration of factors that cause errors. Then the applicability of new methods in MTF reconstruction process is explored in details. With the edge gradient operator introduced in the straight line fitting, an ESF denoising method with a moving window is built to optimize ESF sample points for position refinement on a sub-pixel level. Finally, an attempt to reconstruct MTF using Gaussian function fitting by robust estimation is conducted, and the influences of slant edge angle on accuracy of the MTF measurement are discussed.

\section{MTF Measurement}

Traditional step edge method is confined by the edge angle because of the difficulty to get an ideal edge (along or across the sampling direction) in the image. While the slant edge method reduce the impact of angle, MTF reconstruction is still constrained by the accuracy of edge fitting [5], ESF resampling and LSF fitting, all linked with one another during the reconstruction. High-quality ESF samples requires edge linear fitting with high precision. Since LSF is the derivative of ESF, a slight deviation of ESF can easily cause deformation of LSF. Improvement has been made for all of the three links including sub-pixel positioning of step edge, ESF resampling and LSF fitting to obtain MTF reconstruction of high accuracy.

\subsection{Sub-pixel Positioning of Step Edge.}

High precision edge line fitting requires pinpointing the edge position at the sub-pixel level. Position deviation between the estimated edge points and the real ones is an underlying factor linked to MTF reconstruction error. In order to refine ESF samples, the sub-pixel edge positions are obtained by the mean gradient magnitude method for higher precision after gradient operator is introduced in edge detection. The specific steps are as followed:

- Position the edge points in pixel precision first with edge detection algorithm line by line; 
- Calculate the gradient magnitude $G(x, y)$ of those pixel edge positions along the gradient direction;

- Adjust the edge positions in sub-pixel level in the gradient direction using the gradient component $G_{x}$ and $G_{y}$ as the following formula:

$$
\begin{aligned}
\Delta d_{x} & =\sum_{i=1}^{n} G_{x_{i}} d_{x_{i}} / \sum_{i=1}^{n} G_{x_{i}} \\
\Delta d_{y} & =\sum_{i=1}^{n} G_{y_{i}} d_{y_{i}} / \sum_{i=1}^{n} G_{y_{i}}
\end{aligned}
$$

In the formula, $d_{x_{i}}$ and $d_{y_{i}}$ are the distances between pixels and pixel-level edge positions along gradient direction; $G_{x_{i}}$ and $G_{y_{i}}$ are the gradient components; $n$ is the processed pixel number along the gradient direction.

After sub-pixel edge positions are obtained, the least square fitting is applied under the assumption that the actual edge points lie in one straight line. Figure 1(a) shows the result of least square straight line fitting for the sub-pixel edge positioning. The blue circles in the Figure 1(a) are sub-pixel edge locations and the solid line is the least square fitting line. With edge location confirmed, data points are projected into the gradient direction as shown in Figure 1(b).

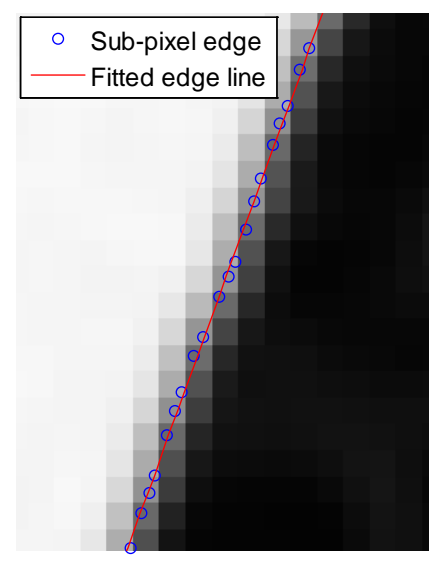

(a)

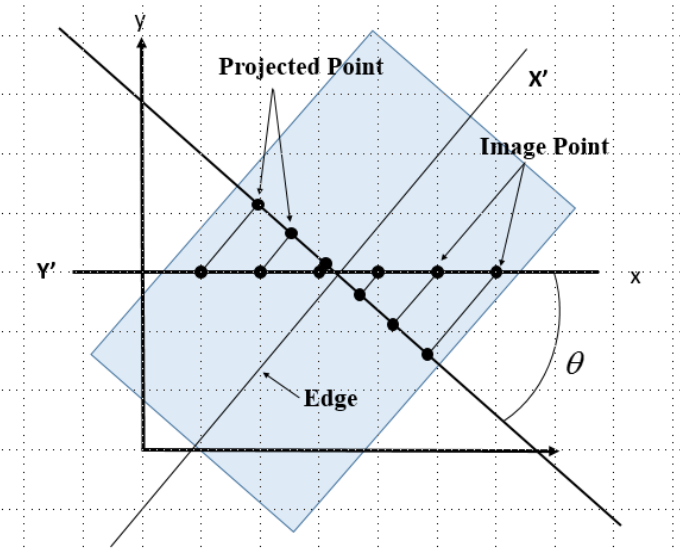

(b)

Figure 1. (a) Edge detection and least square line fitting (b) Projection of ESF samples

\subsection{ESF Denoising and Resampling.}

ESF samples contain gross errors and are unevenly distributed in the local area due to the irregularity of the real scene [6]. The moving window method for denoising and resampling is proposed to minimize the impact of the noise. The window size for denoising is 1 pixel and it moves with 0.05 pixels step. The process is shown in the Figure 2:

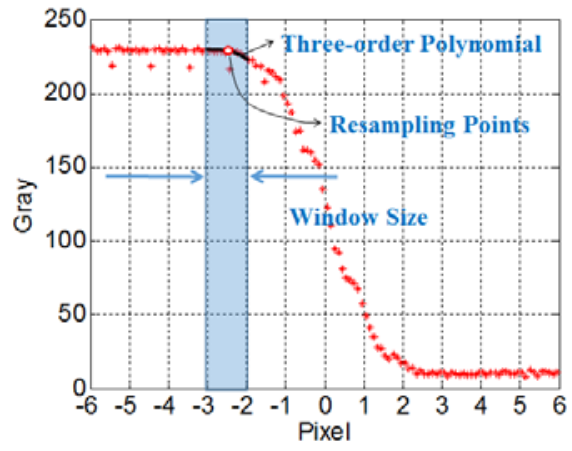

(a)

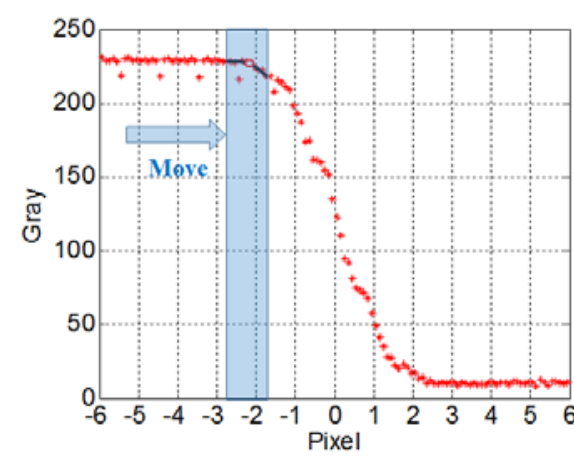

(b)

Figure 2. (a) Demonstration of ESF de-noising (b) Resampling using a moving-window method

To build ESF, the data sampling is often done by nonparametric fitting [7]. Not exactly like the normal approach, during the cubic polynomial fitting process, sample points with large fitting 
residual errors are eliminated in the window and the fitting value at the pixel center is taken as the resampling one. The final ESF are shown in Figure 3(a).

\subsection{Obtaining LSF.}

As is mentioned above, LSF is deduced from the ESF with a derivative filter in the discrete form [8].The Figure 3(b) shows the shape of LSF is approximately Gaussian.

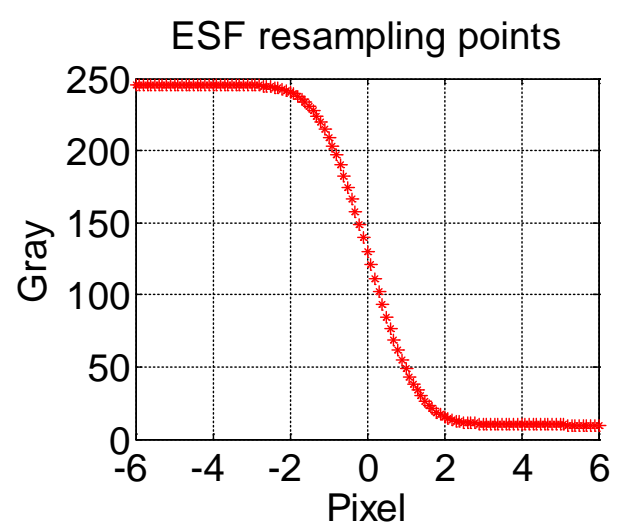

(a)

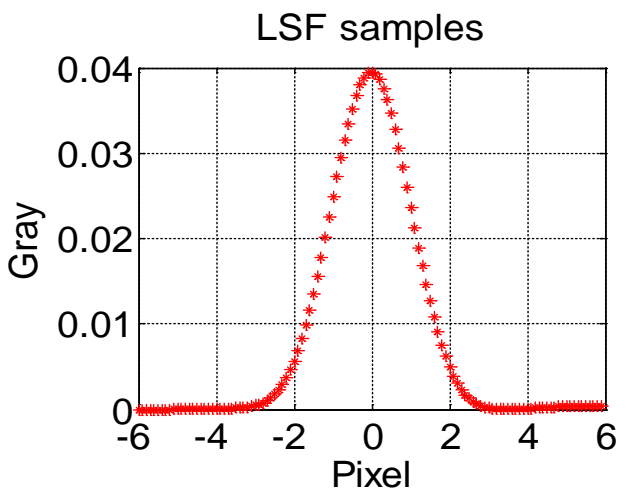

(b)

Figure 3. (a)ESF resampling points (b) LSF samples

\section{Experiments}

Artificial edge images of different angles are added with Gaussian white noise for simulation experiments to assess the accuracy and applicability of the reconstructed MTF by the proposed method. By blurring the artificial edge image with the determinate PSF fitted by Gaussian function, five edge images with degradation are obtained. Wherein the slant angles of the edge in degraded images are set to $0^{\circ}, 15^{\circ}, 22.5^{\circ}, 30^{\circ}, 45^{\circ}$. The standard deviation of the PSF is 1.0.

As can be seen from Figure 4, MTF curves of different data sets almost coincide and are close to that of the theoretically real value, indicating that the method in this article performs well in accuracy and is applicable to slant edges of different angles. It should be noticed that the MTF estimated at the angle $22.5^{\circ}$, closest to the real value, has the highest accuracy as the ESF samples are uniformly distributed. MTF values at the Nyquist frequency are given specifically. Table 1 shows MTF at different frequencies for the five images with different slant edge angles.

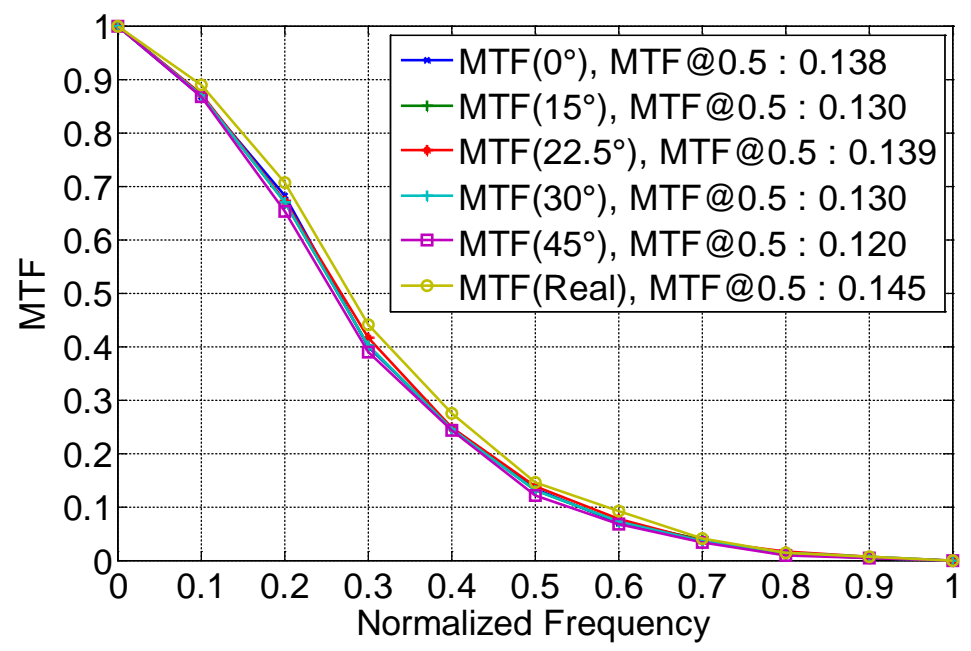

Figure 4. Reconstructed MTF of five images with different edge angles 
Table 1 MTF at different slant edge angles

\begin{tabular}{cccccccccccc}
\hline Frequency & & 0.1 & 0.2 & 0.3 & 0.4 & 0.5 & 0.6 & 0.7 & 0.8 & 0.9 & 1.0 \\
\hline Real Value & & 0.890 & 0.707 & 0.440 & 0.274 & 0.145 & 0.091 & 0.040 & 0.014 & 0.006 & 0.000 \\
& $0^{\circ}$ & 0.868 & 0.682 & 0.399 & 0.248 & 0.134 & 0.069 & 0.037 & 0.013 & 0.003 & 0.000 \\
\multirow{4}{*}{ Angle } & $15^{\circ}$ & 0.869 & 0.670 & 0.402 & 0.245 & 0.130 & 0.072 & 0.037 & 0.012 & 0.003 & 0.000 \\
& $22.5^{\circ}$ & 0.873 & 0.672 & 0.416 & 0.248 & 0.139 & 0.076 & 0.036 & 0.015 & 0.005 & 0.000 \\
& $30^{\circ}$ & 0.869 & 0.670 & 0.402 & 0.246 & 0.130 & 0.071 & 0.035 & 0.013 & 0.006 & 0.000 \\
& $45^{\circ}$ & 0.867 & 0.652 & 0.389 & 0.243 & 0.120 & 0.066 & 0.034 & 0.009 & 0.004 & 0.000 \\
\hline
\end{tabular}

\section{Summary}

The traditional step edge method is limited by the edge angle and it is also sensitive to noise when applied in practice. An improved method is built in this article to reconstruct MTF of the blurred images precisely based on the slant step edge. First of all, data points are projected into the gradient direction after being adjusted by the gradient operator. In addition, as original sample points are in discrete form, the sub-pixel edge positions can be easily affected by the fluctuation of point positions. To tackle this problem, more refined ESF samples are obtained by denoising with a moving window and resampling to alleviate the effect. Experiments demonstrate that the proposed method has better performance with different slant edge angles than the traditional one both in accuracy and feasibility.

\section{Reference}

[1]. Nugent P W, Shaw J A, Kohoe M R, et al. Measuring the Modulation Transfer Function of an Imaging Spectrometer with Rooflines of Opportunity [J]. Optical Engineering.Vol.49 (2010) No.10, p.103201.

[2]. Rangaswamy M K: Two-dimensional On-orbit Modulation Transfer Function Analysis Using Convex Mirror Array [D]. Master of Science. South Dakota State University, the United States .2003.p.30-69.

[3]. L.Helder D, Choi T: IKONOS Satellite in Orbit Modulation Transfer Function (MTF) Measurement Using Edge and Pulse Method [D]. Master of Science. South Dakota State University, the United States .2002.p.4-12.

[4]. Saunier S, Goryl P, Chander G, et al. Radiometric, Geometric, and Image Quality Assessment of ALOS AVNIR-2 and PRISM Sensors [J]. IEEE Transactions on Geoscience \& Remote Sensing.Vol.48 (2010) No.10, p. 385 5-3866

[5]. Chi Y F, Ping Z C, Feng C L. Simulation of In-orbit Satellite Image MTF Measurement Using Edge Method [J]. Imaging Technology. Vol.49 (2007) No.10, p.33-39.

[6]. FAN Chong, LI Guanda, WU Chaoyun, et al. High Accurate Estimation of Point Spread Function Based on Improved Reconstruction of Slant Edge [J]. Acta Geodaetica et Cartographica Sinica.Vol.44 (2015) No.11, p. 1219-1226.

[7]. QIN Rongjun, GONG Jianya. A Robust Method of Calculating Point Spread Function from Knife-edge without Angular Constraint in Remote Sensing Images [J].Journal of Remote Sensing. Vol.15 (2011) No.5, p. 901-907.

[8]. WANG Qiaojue, XU Yuanjing1, YUAN Qiangqiang, et al. Restoration of CBERS-02B Remote Sensing Image Based on Knife-edge PSF Estimation and Regularization Reconstruction Model [C]. 2011 Fourth International Conference on Intelligent Computation Technology and Automation. Washington, DC, USA, 2011, p. 687-690. 